\title{
Methods of low-pressure cylinders throughput improvement for construction of ultra-high capacity generation units
}

\author{
A. Zaryankin, A. Rogalev, I. Garanin \& S. Osipov \\ Moscow Power Engineering Institute, Russia
}

\begin{abstract}
State-of-the art power plant designs are based on generating unit capacities of 1200 MW. Innovative low-pressure (LP) cylinder designs with increased throughput are viewed among key approaches to further capacity improvement. This paper is specifically concerned with improving LP throughput.

Supported by computations, this paper examines three throughput-maximizing LP cylinder concepts enabling the throughput of a single outlet to be increased by $40 \%$, thereby raising unit power capacity to $1800-2000 \mathrm{MW}$. These include a cylinder with a $1400 \mathrm{~mm}$ final-stage blade, a cylinder with flow reverse downstream of penultimate stage and a two-tier LP cylinder. Each of these designs carries with it a distinctive combination of advantages and shortcomings.

A two-tier LP cylinder is shown by computations to be the best approach. It features best economy while providing the necessary strength. The use of a twotier pass-through section in place of classical section enables the outlet section area to be increased from $11.3 \mathrm{~m}^{2}$ to $16.3 \mathrm{~m}^{2}$ while using $1200 \mathrm{~mm}$ long final stage blades in both cases. In this case, LP cylinders operate with an efficiency of $84 \%$ and the maximum turbine capacity may reach $2000 \mathrm{MW}$.

The use of LPCs with increased throughput allows us to construct new power units with ultra-high capacity and to improve the efficiency indicators of the units of the existing power range. Both of these directions contribute to the achievement of steady state of the energy sector by improving the ecology efficiency.

Keywords: low-pressure cylinder, steam turbine, two-tier cylinder.
\end{abstract}




\section{Introduction}

The problem of increasing unit power is of great practical interest for power units as lower use of metal in this case translates into reduced capital expenditures per megawatt.

The standard approach to increasing unit capacity is based on increasing stream throughput in the flow-through part of the turbine. The throughput of the final lowpressure (LP) cylinder stages is a constraint on steam throughput capacity.

Our examination of potential approaches for increasing steam turbine throughput will involve equation (1) that describes capacity constraint:

$$
N \approx \sum F_{\mathrm{a}} \cdot \frac{M_{c_{2 a}} \cdot p_{2}}{q}
$$

where, $\Sigma F_{a}-$ total outlet section area $\left(\mathrm{m}^{2}\right)$;

$M_{C 2 a}$ - Mach number of the flow component of the velocity of steam leaving the blade passage of the final stage;

$p_{2}$ - outlet pressure $(\mathrm{Pa})$;

$q-$ specific flow rate of steam let into the condenser $(\mathrm{kg} /(\mathrm{kW} \cdot \mathrm{h}))$. This value depends mainly on initial steam parameters.

As equation (1) shows, LP cylinder power depends on a number of factors, nevertheless increasing the total outlet area is the only way forward with state-ofthe-art technology. Among other factors, Mach numbers deserve a closer look. Modern turbine hoods feature outflow speeds close to limit values -0.75 at present. If the speed were increased further, the outlet hood of LP cylinder will cause greater drag, reducing cylinder efficiency. Therefore, it is inexpedient to increase capacity by opting for a higher $M_{C 2 a}[1]$.

An increase in final pressure would degrade the overall economy of the turbine unit. For example, a change from $p_{2}=3.5 \mathrm{kPa}$ to $p_{2}=4 \mathrm{kPa}$, final stage dimensions remaining the same, will increase turbine rated power by $13 \%$ approximately, while unit efficiency for high-performance turbine will decline by an order of $0.5 \%$ [2]. Consequently, increasing outlet cross-section in the most promising approach to achieving greater turbine capacity.

The problem of increasing flow rate by opting for a greater total output crosssection may be solved either quantitatively by increasing the number of outlets or qualitatively by increasing LP cylinder throughput while maintaining the same number of steam outlets to the condenser. The present paper is concerned with the second, qualitative approach to the problem, namely looking for ways to increase the frontal outlet cross-section area of the LP cylinder.

Various research institutes tried to address this problem in a number of ways. For example, the Central Boiler and Turbine Institute came up with a cylinder concept [3] featuring flow reverse.

The same problem was featured in a series of patents and publications by researchers from Moscow Power Engineering Institute and TurboZAR CJSC [48]. These works were based on the Schellens stage [9] used as a base for the new two-tier high-throughput LP cylinder. 
The expediency of a semidouble exhaust is quite convincingly illustrated by comparing potential options for increasing the working throughput [10]. However the two-tier LP cylinder was not considered among options in the work cited above.

The present work is specifically concerned with comprehensive treatment of all advantages and disadvantages of increasing the frontal cross-section of LP cylinder outlet.

\section{LP cylinder with a $1400 \mathrm{~mm}$ long final-stage blade}

One of the most obvious approaches to increasing LP cylinder throughput involves longer final-stage blades. Increased length of final-stage blades translates into greater outlet cross-section which means greater throughput capacity at the same flow velocity.

High-RPM steam turbines of the 300-800 MW range are mostly using $960 \mathrm{~mm}$ final stage blades. $1200 \mathrm{~mm}$ blades have emerged as a result of designers striving for increased capacity without adding more cylinders.

To enable a detailed analysis of all advantages and disadvantages inherent in this method, aerodynamic and strength computations have been carried out for LP cylinder blading, leading to a design with $1400 \mathrm{~mm}$ long final-stage blades (fig. 1).

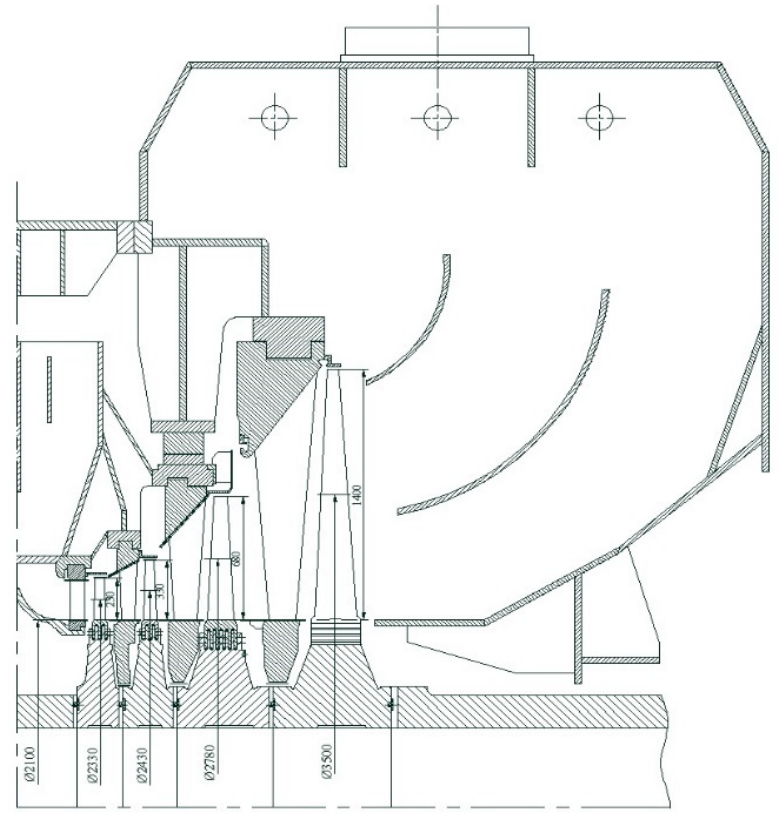

Figure 1: Sketch of a single low-pressure cylinder flow with a $1400 \mathrm{~mm}$ final stage. 
The transition from $1200 \mathrm{~mm}$ blades to $1400 \mathrm{~mm}$ blades will enable the axial outlet area of a single flow to be brought up from $11.3 \mathrm{~m}^{2}$ to $15.4 \mathrm{~m}^{2}$. Computations have been made for design parameters representative of LP cylinders used in condensation power plant turbines of $300-1200 \mathrm{MW}$ power range $\left(P_{0}=0.31 \mathrm{MPa}\right.$, $\left.t_{0}=231^{\circ}, P_{k}=3.5 \mathrm{kPa}\right)$. In order to determine the average stage diameter, the inverse divergence parameter (indicating divergence losses in the stage) must be known. By analysing geometrical properties of final-stage blades in high-capacity turbines [11] this parameter was estimated in the range $2.5<\theta<3$ for turbine blades longer than $1200 \mathrm{~mm}$. An estimate of 2.5 was used for our LP cylinder design predetermining the average diameter of $3.5 \mathrm{~m}$ and root diameter $2.1 \mathrm{~m}$ which was assumed to be constant throughout the entire blading section. Root diameters of $1.6 \mathrm{~m}$ for $960 \mathrm{~mm}$ blades and $1.8 \mathrm{~mm}$ for $1200 \mathrm{~mm}$ blades are used in existing LP cylinders of high-capacity power plant units. As a consequence of the move toward greater diameters, greater temperature drop has per stage has become necessary to maintain optimum $u / c f$ ratios, therefore a cylinder with longer final-stage blades designed for similar parameters will have with fewer stages. Cylinder decomposition analysis has identified 4 as the optimum number of LP cylinder stages for $1400 \mathrm{~mm}$ long final-stage blades, even though similar cylinders with 960 and $1200 \mathrm{~mm}$ blades are made in five-stage versions.

Last stage efficiency stands at just $71 \%$. Such a low efficiency mostly results from large residual velocity losses. Residual velocity losses at fixed axial section area mostly depend on the velocity i.e. Mach number. The maximum Mach number (0.75) was chosen to maximize potential flow rate.

Additional losses due to divergence, humidity and associated losses bring efficiency down to $55 \%$. Moreover, the use of long vanes with just four stages in the cylinder results in extremely high opening angles (more than $45 \%$ ) in the blading section, depressing efficiency even further. A realistic expectation of efficiency for such a stage would be about $50 \%$.

The overall efficiency of the cylinder turns out to be quite low averaging about $80 \%$. Low overall efficiency of the cylinder woes itself to extremely low efficiency of the final stage. Four-stage design of the cylinder burdens this stage with excessive enthalpy drop. For example, an enthalpy drop of $203 \mathrm{~kJ} / \mathrm{kg}$ is available at this stage with $960 \mathrm{~mm}$ long vanes, rising to $260 \mathrm{~kJ} / \mathrm{kg}$ with $1400 \mathrm{~mm}$ long vanes. This increased enthalpy drop is a necessary consequence of having to maintain $u / c f$ rate close to the optimum one. This means that a low-economy stage will have to draw down additional 50 to $60 \mathrm{~kJ} / \mathrm{kg}$ that could have been utilized efficiently at other stages. A conclusion can be drawn therefore that an attempt to increase LP cylinder throughput by opting for longer vanes definitely degrades economy.

Strength becomes a consideration apart from economy. Computations have prompted the conclusion that the final stage of LP cylinder with $1400 \mathrm{~mm}$ long vanes will operate in critical conditions, meaning that safety factor for tensile stresses will stand at just 1.3 if titanium is used. Most assemblies of powergenerating equipment must be designed with a safety factor of at least two. It should be further noted that longer vanes with greater root diameters definitely cause peripheral circumferential velocities to increase, intensifying erosion and 
droplet-induced wear of final-stage vanes. A $1400 \mathrm{~mm}$ final-stage blade will experience circumferential velocities of $725 \mathrm{~m} / \mathrm{s}$ at its tips - more than $100 \mathrm{~m} / \mathrm{s}$ greater than in cylinders with $1200 \mathrm{~mm}$ long blades. Considering that the rate of erosive droplet wear is a function of the peripheral velocity squared, such a difference may shorten the useful life of the final stage [11].

Strength computations support the conclusion that the quality of state-of-theart structural materials used in power-generating equipment falls short of the requirements posed by $1400 \mathrm{~mm}$ long blades. In addition, aerodynamic computations point to low efficiency of the final stage, rendering the entire cylinder less economic.

\section{LP cylinder with flow turnaround}

Longer final-stage vanes are not the only option for increasing LP cylinder throughput. A greater outlet area can also be achieved by expanding frontal opening area by means of two-tier stages with a semidouble exhaust flow pattern. This approach was pioneered in the Baumann exhaust design where the flow is divided into two parts upstream of the final stage. This design is used in Russian K-200-130 turbines. A lengthwise section view of a single flow from double-flow LP cylinder with Baumann exhaust design is shown in fig. 2. A part of the stream in the top tier of the stage is expanded to condenser pressure while the other part, after expanding in the bottom tier, passes to final LP cylinder stage.

LP cylinders with Baumann exhaust failed to gain traction as this design results in a severe drop in internal relative efficiency of the LP cylinder. This drawback mainly stems from the top-tier enthalpy drop at penultimate stage being equal to a

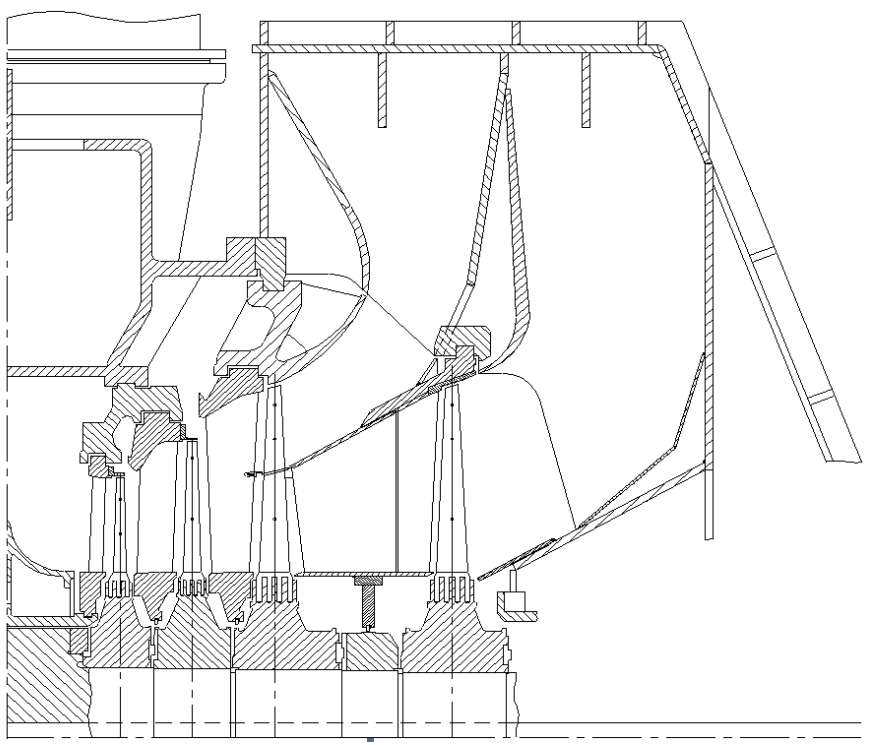

Figure 2: $\quad$ Low-pressure cylinder with Baumann exhaust. 
sum total of enthalpy drops on bottom tier and at the final stage of the turbine. As a consequence, the top tier works at a velocity ratio $u / c f$ below optimum while experiencing greater supersonic velocities relative to the bottom-tier velocities.

The need to do away with above-mentioned deficiencies of Baumann exhaust has resulted in a blading section concept that separates a part of the flow and rotates it by $180^{\circ}$ downstream of penultimate stage. A sketch of LP cylinder with this blading design is shown in fig. 3 .

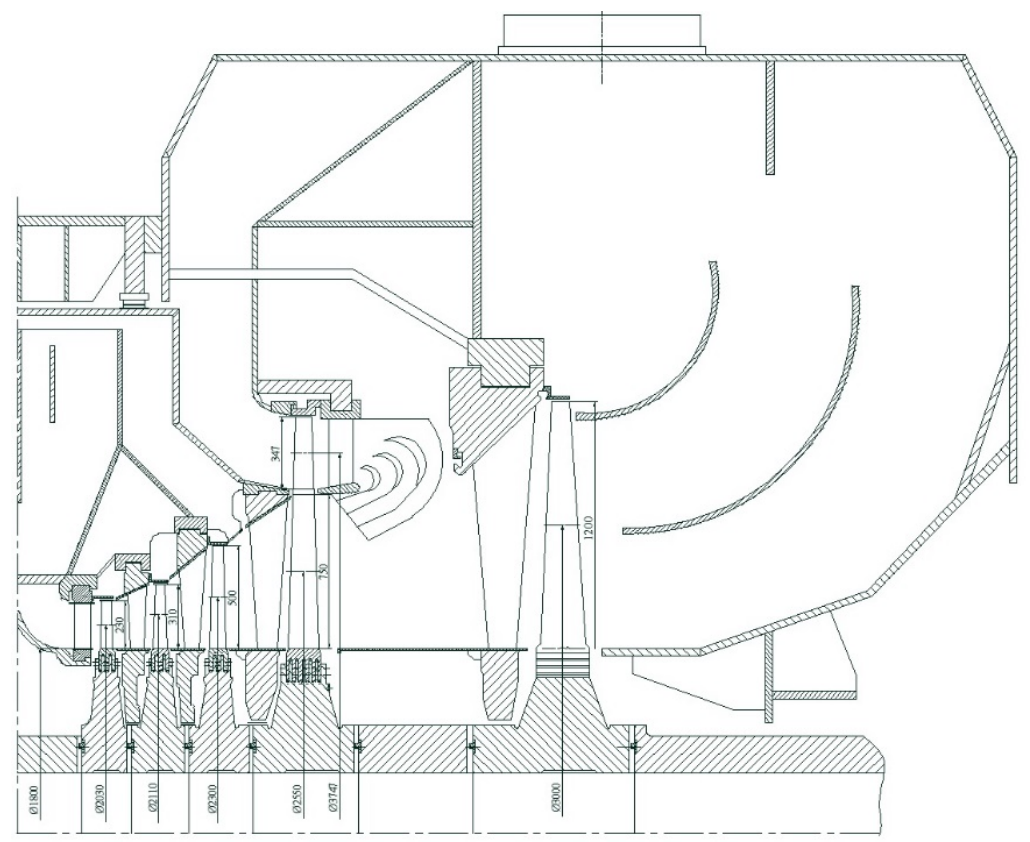

Figure 3: Sketch of a single low-pressure cylinder with $180^{\circ}$ flow turnaround.

Flows of the working medium are structured as follows: steam leaving the bottom tire of the penultimate two-tier stage is divided into two flows one of which is directed to the final stage and the other is turned by $180^{\circ}$ to come to the upper tier at penultimate stage. This solution shifts the burden of enthalpy drop toward the second tier of the penultimate stage where it would become equal to the enthalpy drop at the final stage of the turbine. However the need to tap steam flow and then rotate it by $180^{\circ}$ raises extreme difficulties.

Such an approach increases the total frontal cross-section area of a single exhaust by $16.3 \mathrm{~m}^{2}$ at final-stage vane length of $1200 \mathrm{~mm}$. Such vanes are customary in turbine designs have been in production for a long time. This choice of vane length enables the root diameter to be maintained at $1.8 \mathrm{~m}$. If designed for the same steam parameters as the cylinder with $1400 \mathrm{~mm}$ long vanes, a cylinder featuring flow rotation with $1200 \mathrm{~mm}$ long final-stage vanes would have a fivestage structure with a two-tier penultimate stage. 
The need to introduce steam turnaround - specifically the presence of a rotation chamber - causes the energy of the outlet velocity at penultimate stage to be lost completely. This explains a 5\% drop in vane efficiency at fourth-stage LP cylinder compared to cylinders without a rotation chamber. Moreover, flow structure becomes severely irregular upon entry to the second tier. Special turning devices installed inside the rotation chamber to equalize the velocity profile result in additional energy losses associated with flow rotation, therefore any design that involves flow rotation - irrespective of the actual device chosen for this job - will cause greater losses than an alternative without flow rotation.

Computations indicate that the design with flow separation and rotation by $180^{\circ}$ will have a capacity-averaged LP cylinder efficiency of $82 \%$. This value can be viewed as an "optimistic" estimate of cylinder efficiency as computations assumed that flow would be rotated without upsetting the flow structure. Vortices will inevitably appear in the flow as it makes a turn, decreasing the efficiency of the top tier at penultimate stage. In addition, vortex formation will "clog" a part of the opening area, decreasing the maximum potential steam flow in the cylinder as a consequence.

The application of a solution involving separation and rotation of a part of the total flow will improve the efficiency of final stages at top and bottom tiers. The frontal area of such a cylinder will exceed the outlet cross-section area of a cylinder with $1400 \mathrm{~mm}$ long vanes by $0.9 \mathrm{~m}^{2}$. In turn, it allows to decrease axial velocity while maintaining the flow rate, recouping $32.6 \mathrm{~kJ} / \mathrm{kg}$ in residual velocity losses thereby improving the internal relative efficiency of the final stage to $65 \%$. This efficiency is almost on $10 \%$ higher than that of a final stage with $1400 \mathrm{~mm}$ long vanes. The second tier has the greatest divergence conferring an internal efficiency of about $76 \%$. Another design of LP cylinder blading similarly produces significant changes in strength conditions of the final two stages. Strength computations have revealed the penultimate stage rather that the ultimate stage to bear the greatest load. The addition of the second tier requires stage length to be increased, placing additional tensile stresses onto the fourth stage of the cylinder. However a replacement of high-alloy steel with VT-6 Russian titanium brand has made it possible to maintain the strength safety factor for the penultimate stage at an acceptable level (not lower than 1.8).

The use of a $1200 \mathrm{~mm}$ long vane at the final stage with a diameter of $1.8 \mathrm{~m}$ makes it possible to maintain a circumferential velocity of about $660 \mathrm{~m} / \mathrm{s}$, ensuring reliable operation from the standpoint of droplet-induced corrosion of rotor vanes.

The original approach to a semidouble exhaust by turning the working medium downstream of penultimate stage solves the problem of ensuring final stage reliability as ample exhaust cross-section becomes available without having to abandon well-tried vane length. However, the unsolved problem of designing a turning chamber with energy losses acceptably small weighs down on cylinder economy. 


\section{Two-tier LP cylinder}

A promising solution for the problem of increasing LP cylinder throughput lies in the mastering of multiple-stage two-tier blading designs where the upper stage would comprise a vane assembly totally independent of the bottom tier - with an individual optimum choice of vane steps, profiles and numbers. A sketch of a single LP cylinder flow section design is shown in fig. 4. This cylinder was designed for the same parameters and flow rates as described above.

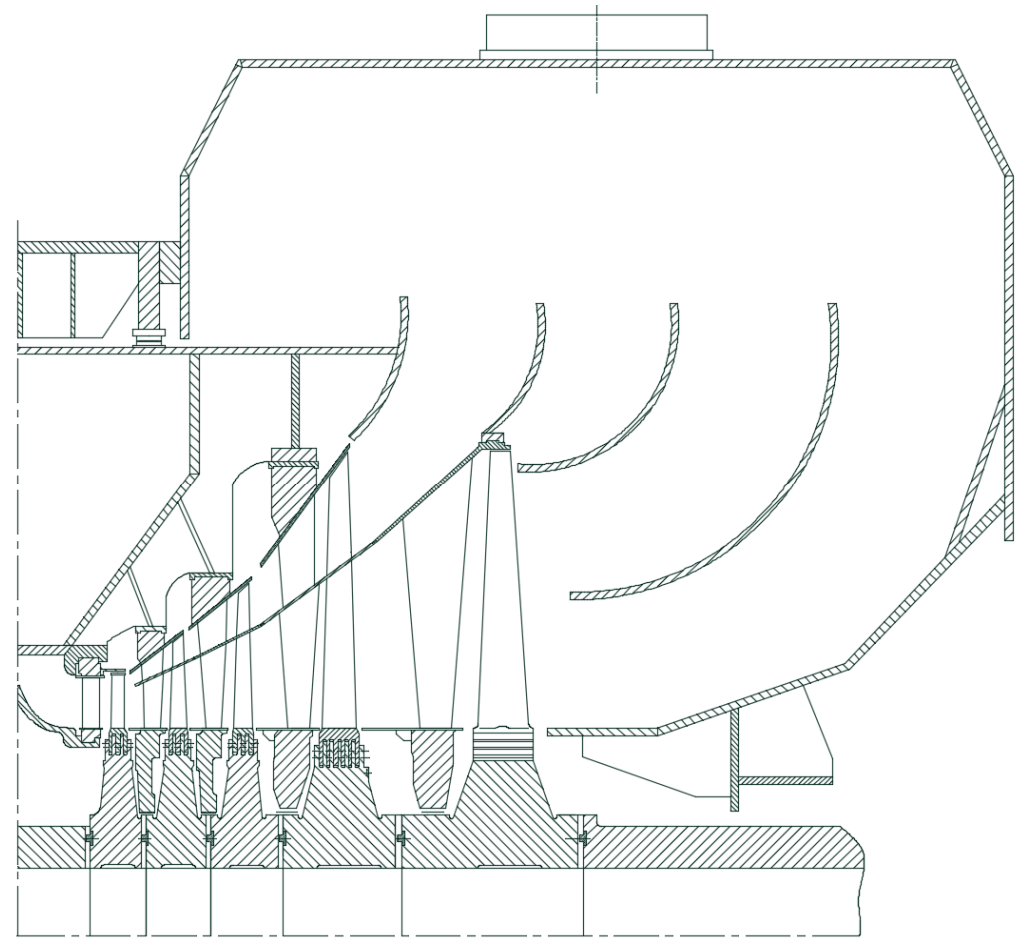

Figure 4: Two-tier low-pressure cylinder structure.

Steam flow is arranged in the following manner: downstream of the first stage the flow is divided: its greater part continues to expand in the four stages of the bottom tier while the other part is expanded to condenser pressure in three top-tier stages. A smaller number of second-tier stages necessary to expand steam from the pressure downstream of LP cylinder stage one to the condenser pressure is explained by the fact that median dimensions of the second tier are greater than those of the bottom tier, therefore higher enthalpy drops may be realized at toptier stages than on bottom-tier stages.

This solution retains specific benefits of LP cylinder designs with flow rotation. The use of a $1200 \mathrm{~mm}$ long vane makes it possible to retain the same opening 
angle of the blading section. Greater exhaust area facilitates reduction of outlet velocity thereby bringing down residual velocity losses and improving the efficiency of final stages as a consequence. Being able to dispense with rotation chamber is another notable benefit.

The total efficiency of the two-tier LP cylinder design was estimated at $84 \%$. For comparison, a LP cylinder with $1400 \mathrm{~mm}$ long final-stage vanes will have an efficiency of $80 \%$ while the rotated-flow concept will feature an efficiency of $82 \%$.

Improved efficiency is a benefit of isolating a part of the flow to expand in the upper tier where reduced vane height and greater root diameter eliminate divergence losses altogether, making internal efficiency of second-tier stages exceed that of bottom-tier stages. Fig. 5 shows the impeller disc of the penultimate stage.

A specific issue with two-tier LP cylinder designs is a leakage of steam from the peripheral zone of the bottom tier into the root zone of the top tier. Computations indicate that the total leakage may reach $3 \%$ of the steam flow at cylinder inlet unless additional seals are used. Consequently, additional inter-tier seal systems must be designed. A sealing system may inhibit leaks, bringing total leakage rates to $1 \%$ of incoming steam flow.

The transition to two-tier LP cylinder designs brings about higher tensile stresses at the penultimate (two-tier) stage. This means that the steel normally used for the penultimate stage no longer meets strength criteria. Therefore a penultimate stage made of titanium was used in these computations. This change ensures an adequate strength safety factor (1.8 or greater).

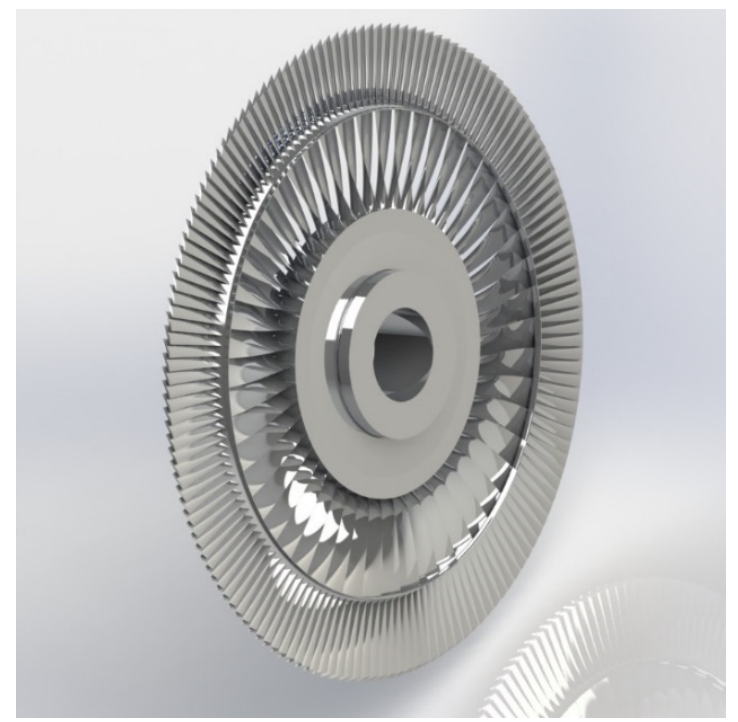

Figure 5: Two-tier impeller wheel disc of penultimate LP cylinder stage. 


\section{The prospects of the use of the new two-tier cylinder in terms of energetics' sustainable development}

Increased throughput is the key to the new level of high-speed steam turbine unit capacity. Construction of ultra-high power units allows to reduce relative capital investments and in this way increases economic efficiency of the power plant.

The use of a two-tier cylinder for power units of already mastered capacity with maintaining of the number of cylinders raises the efficiency of power production. Increasing of the exhaust's axial area leads to reduction of the exhaust speed, and, therefore, of the losses in the last stage. Moreover, the reduced output speed makes possible some additional reduction of the condenser pressure. Wherein the whole turbine efficiency raises by $0.7 \%$ that increases efficiency of the power plant by $0.3 \%$. The improvement of the power production efficiency has positive effect on emissions of $\mathrm{CO}_{2}$ into the atmosphere. In case of a coal-fired power unit with capacity of $800 \mathrm{MW}$, the use of the new cylinder allows to reduce carbon dioxide emissions by $1.302 * 10^{7} \mathrm{~m}^{3}$ a year.

\section{Conclusions}

This paper was concerned with a comparative survey of three potential approaches to increasing LP cylinder throughput. Key features of designed solutions are summarized in table 1 .

Table 1: A comparison of LP cylinder throughput improvement concepts.

\begin{tabular}{|l|c|c|l|l|}
\hline Designation & Exhaust area & Efficiency & Key advantages & Disadvantages \\
\hline $\begin{array}{l}\text { LP cylinder } \\
\text { with 1400 } \\
\text { mm long } \\
\text { final-section } \\
\text { vane }\end{array}$ & 15.4 & $80 \%$ & Few stages & $\begin{array}{l}\text { Low efficiency, } \\
\text { final stage } \\
\text { strength issues }\end{array}$ \\
\hline $\begin{array}{l}\text { LP cylinder } \\
\text { with flow } \\
\text { rotation }\end{array}$ & 16.3 & $82 \%$ & $\begin{array}{l}\text { Increased } \\
\text { thriciency, high } \\
\text { throughput }\end{array}$ & $\begin{array}{l}\text { Losses for } \\
\text { rotation, length }\end{array}$ \\
\hline $\begin{array}{l}\text { Two-tier LP } \\
\text { cylinder }\end{array}$ & 16.3 & $84 \%$ & $\begin{array}{l}\text { Highest } \\
\text { efficiency, high } \\
\text { throughput }\end{array}$ & $\begin{array}{l}\text { Large number } \\
\text { of stages }\end{array}$ \\
\hline
\end{tabular}

The low-pressure cylinder with final-stage vane is clearly inferior to the twotier LP cylinder both in terms of efficiency (4\% drop in efficiency factor) and in terms of reliability. The two-tier LP cylinder is based on tried $1200 \mathrm{~mm}$ titanium vanes while the cylinder with $1400 \mathrm{~mm}$ long final-stage vanes is based on yet untested vane design that calls for new structural materials (VT-6 titanium provides a strength safety factor of just 1.3). 
Flow turnaround enables the exhaust section area to be increased while avoiding vane lengths greater than $1200 \mathrm{~mm}$. However this solution faces significant shortcomings. Such a cylinder will have greater overall efficiency than the long-vane $(1400 \mathrm{~mm}) \mathrm{LP}$ cylinder design. However the addition of a rotation chamber decreases fourth-stage efficiency and increases rotor length potentially degrading vibration performance.

To summarize, the two-tier design appears to be the most promising way of increasing LP cylinder throughput. It is devoid of all shortcomings inherent in alternate designs. Peculiar issues with two-stage LP cylinder design include having to deal with a large number of stages and cross-flows between tiers. On balance, exceptional economy and reliability make those deficiencies tolerable.

Throughput improvement with a combination of high performance and adequate reliability is clearly only possible with two-tier cylinder designs.

The use of the best way of the throughput increasing, the two-tier cylinders were proved to be, makes it possible to construct power units of previously unavailable capacity (up to $2000 \mathrm{MW}$ ) and to increase the efficiency indicators of already existing equipment, that promotes the further sustainable development of the power industry.

\section{Acknowledgements}

The research has been carried out in Moscow Power Engineering Institute with financial support from the Russian Federation represented by Ministry of Education and Science of the Russian Federation under Agreement No. 14.577.21.0072 on Grant Provision dated June 5, 2014, for the purpose of implementation of the Federal Target Program "Research and Development in Priority Growth Fields of Russian Science and Technology Sector for 2014-2020". The unique ID of applied research: RFMEFI57714X0072.

\section{References}

[1] Zaryankin, A.E., Simonov, B.P. Exhaust hoods of steam and gas turbines, Moscow: MPEI, 2002.

[2] Shcheglyayev, A.V. Steam turbines: the theory of the thermal process and turbine design, sixth ed., Mosenergoatomizdat: Moscow, 1993.

[3] Nishkevich, V.I., Bakuradze, M.F., Safonov, L.P., Khrabrov, P.V. Analysis and application prospects of two-tier exhausts in flowing parts of highpower steam turbines low-pressure parts, TsKTI: Leningrad, pp. 46-59, 1978.

[4] Zaryankin, A.E., Zaryankin, V.A., Arianov, A.E., Rogalev, A.N., Chukin, A.I. Patent RU 139602 C1, 2013.

[5] Zaryankin, A.E, Arianov, S.V., Zaryankin, V.A. Patent RU 2372491 C2, 2007.

[6] Zaryankin, A.E, Arianov, S.V. Patent RU 2378516 C2, 2008.

[7] Zaryankin, A.E., Arianov, S.V., Zaryankin, V.A. Patent RU 2296224 C1, 2006. 
160 Energy and Sustainability VI

[8] Zaryankin, A.E., Arianov, S.V., Zaryankin, V.A. Patent RU 2306424 C1, 2006.

[9] Schellens, C.A. Patent US 1242246 A, 1916.

[10] Filippov, G.A., Nazarov, O.I., Tomkov, Yu.P., Lisyansky, A.S. Key research directions and prospects for designing 1500-1700 MW nuclear power plant turbine installations. VTI: Moscow, 2002.

[11] Kostyuk, A.G., Frolov, V.V., Bulkin, A.Yu., Trukhniy, A.D. Steam and gas turbines for thermal power plants, Moscow: MPEI, 2008. 